\title{
Evaluation of Bacterial Contamination in Clinical Environment of Sari Dental School in 2018
}

\author{
N Hoshyari' ${ }^{1}$, Z Allahgholipour ${ }^{2}$, M Ahanjan³ ${ }^{3}$, M Moosazadeh ${ }^{4}$, \\ M Zamanzadeh $5^{\star}$
}

1. Assistant Professor, Department of Endodontics, Faculty of Dentistry, Mazandaran University of Medical Sciences, Sari, Iran

2- Dentist, Sari, Iran

3. Associate Professor, Department of Medical Microbiology and Virology, Molecular and Cell Biology Research Center, Mazandaran University of Medical Sciences, Sari, Iran

4- Assistant Professor, Health Sciences Research Center, Addiction Institute, Mazandaran University of Medical Sciences, Sari, Iran

5- Assistant Professor, Department of Oral and Maxillofacial Pathology, Faculty of Dentistry, Mazandaran University of Medical Sciences, Sari, Iran

\begin{tabular}{|c|c|}
\hline & ABSTRACT \\
\hline $\begin{array}{l}\text { ARTICLE INFO } \\
\text { Article History } \\
\text { Received: Nov } 2018 \\
\text { Accepted: Dec } 2018 \\
\text { ePublished: Jan } 2019 \\
\end{array}$ & $\begin{array}{l}\text { Background and Aim: Bacterial contamination of clinical surfaces of dental units that } \\
\text { have been touched or been exposed to patients' blood or saliva can be a reservoir for } \\
\text { infections, leading to cross-contamination. This study aimed to evaluate bacterial con- } \\
\text { tamination in the clinical environment of Sari Dental School in } 2018 \text {. } \\
\text { Materials and Methods: In this cross-sectional (descriptive-analytical) study, }\end{array}$ \\
\hline $\begin{array}{l}\text { Corresponding author: } \\
\text { Assistant Professor, } \\
\text { Department of Oral and } \\
\text { Maxillofacial Pathology, } \\
\text { Faculty of Dentistry, } \\
\text { Mazandaran University } \\
\text { of Medical Sciences, } \\
\text { Sari, Iran E-mail: } \\
\text { zamanzadehmaryam@ } \\
\text { gmail.com }\end{array}$ & $\begin{array}{l}\text { samples were randomly collected from } 15 \text { active dental units of five departments of } \\
\text { Sari Dental School, including surgical, pediatrics, prosthodontics, endodontics, and re- } \\
\text { storative dentistry departments. Samples were collected from headrests, light handles, } \\
\text { and dental seats using moist sterile swabs, and air samples were collected using agar } \\
\text { plates. Sampling was carried out before and after dental practice. The samples were } \\
\text { transferred to the microbiology laboratory to determine the number of various microor- } \\
\text { ganism colonies. Data were analyzed using Chi-square, McNemar, and Kruskal-Wallis } \\
\text { tests. P-values lower than } 0.05 \text { were considered significant. } \\
\text { Result: A significant difference was found between the frequency of contamination be- } \\
\text { fore and after clinical practice based on McNemar test results. Staphylococci were more } \\
\text { prevalent on the surfaces. Kruskal-Wallis test revealed no significant difference in the } \\
\text { total number of microorganisms between different departments after dental practice. } \\
\text { Bacterial contamination of air was greater than other parts, followed by dental seats. } \\
\text { Conclusion: Microbial contamination of dental units considerably increases after treat- } \\
\text { ment of each patient. Therefore, disinfection of dental unit surfaces and seats between } \\
\text { each patient is essential. Also, methods of infection control must be supervised to pre- } \\
\text { vent cross-infection. } \\
\text { Keywords: Equipment Contamination, Dental Infection Control, Disinfection, Micro- } \\
\text { organism }\end{array}$ \\
\hline
\end{tabular}

J Res Dent maxillofac Sci. 2019; 4 (2) :19-25 DOI: 10.29252/jrdms.4.2.19

\section{Introduction:}

Today, infection control is considered as a critical factor in dental care. According to the literature, inappropriate disinfection of dental environments can lead to the transmission of infectious diseases and subsequent contamination; this is a threat to the health of dental staff and patients. Infectious diseases in a dental environment may spread from patients to dental staff, from staff to patients, from one patient to other patients, and from dental office to the community. ${ }^{(1)}$ 
Infectious diseases can spread through direct or indirect contact, blood or oral secretions, aerosols, and contaminated equipment. ${ }^{(2)}$

With the increase in the number of infectious diseases, transmissible through blood and saliva, dentists play an important role in reducing this risk by following the rules and principles of proper disinfection.

Routine infection control includes maintenance of hand hygiene, disinfection, and contact isolation to prevent hospital infections. Accumulation of pathogenic microorganisms on the surface of various objects in a dental office, such as dental chairs, units, headlamps, suction tips, and dental instruments and equipment and their transmission to patients, the dentist, and dental staff lead to cross-infection. ${ }^{(1)}$ The equipment and surfaces of a dental office are constantly exposed to particles that are contaminated with blood and saliva of patients. Most of the contamination of these aerosols is due to gram-positive cocci (viridans Streptococci and Staphylococci). ${ }^{(3)}$ Clinical surfaces in dental offices are not in direct contact with patients; however, they may become infected during work and then act as a reservoir for microbial contamination. These surfaces can become contaminated directly due to the spread of suspended particles and contact with blood, saliva, and water containing body secretions, or indirectly through contact with contaminated instruments. Lamp handles, unit control keys, seat control keys, headrests, handpiece handle, air-water syringe, and dental seats are examples of clinical surfaces. ${ }^{(4)}$

Proper hand hygiene and the use of personal protective equipment, such as gloves, are important in reducing the potential for transmission of infection through such surfaces although it is more important to use protective covers or clean and disinfect surfaces between patients. These surfaces, if not protected with appropriate covers, should be cleaned after each treatment session. ${ }^{(5)}$ To improve the level of infection control and to increase the quality of clinical conditions, periodic examination of clinical surfaces is very important. By doing so, we can identify the weaknesses in controlling infection in each section and then correct and improve them.
Therefore, given the importance of the issue, the present study examined bacterial con tamination in the clinical environment of Sari Dental School in 2018.

\section{Materials and Methods:}

This study is a cross-sectional (descriptive-analytical) research that was carried out in 2018 to investigate microbial contamination on different clinical surfaces at Sari Dental School.

Samples were prepared from the surfaces of unit headrests, lamp handles, dental seats, and the air surrounding the units in different departments, including surgical, pediatrics, prosthodontics, endodontics, and restorative dentistry departments. According to a study by Valian et al, a sample size of 114 was determined. ${ }^{(5)}$

In the present study, from 50 active units in different departments of Sari Dental School, 15 units were selected according to the sample size. A stratified systematic random sampling was done. Defective and out-of-order units were excluded. Random samples were collected from three units in each of the departments (surgical, pediatrics, prosthodontics, endodontics, and restorative dentistry). For each unit, the samples were collected from four surfaces, including the headrest, lamp handle, dental seat, and the air and the aerosols existing in a radius of one meter around the unit. The sampling was carried out before and after clinical practice. Overall, a total of 120 samples were prepared.

The samples were collected from the mentioned surfaces using wet sterile swabs (dipped in physiological serum) and aseptic tips. ${ }^{(5)}$

Sterilized test tubes containing physiological serum and a swab were used for sampling. Before clinical practice (after disinfection and waiting for 10-15 minutes for the disinfectants to act), the samples were collected using sterilized swabs and through rubbing the swab on intended surfaces in an area with a $10-\mathrm{cm}$ length and a $2-\mathrm{cm}$ width. The swab was placed in a capped tube. After the treatment session, sampling was carried out as described above. ${ }^{(5)}$

Air samples were also prepared using nutrient agar plates. The plates were placed at a distance of 1 meter from the unit headrest and a height of $50 \mathrm{~cm}$ from the ground surface for 30 
minutes such that airborne particles (droplet nuclei, dust particles, bacteria suspended in the air, etc.) lay on the agar surface. ${ }^{(2)}$

Transport media containing sampling swabs and Mueller-Hinton plates were transferred to the microbiology laboratory of the Faculty of Medicine and were placed in an incubator for 24 hours at $37^{\circ} \mathrm{C}$. After 24 hours, the samples were diluted and transferred to blood agar media and eosin methylene blue (EMB). Bacterial counting was performed, and the results were reported in terms of the colony-forming unit (CFU). ${ }^{(5)}$ To determine the type of bacteria, a gram stain slide was prepared. Then, initial diagnostic tests, such as oxidase and catalase, as well as exclusive biochemical tests were performed using the Microgen diagnostic kit (Microgen Bioproducts Ltd., Camberley, UK) for the final detection of bacteria.

In this study, Staphylococcus aureus, Staphylococcus epidermidis, Staphylococcus saprophyticus, viridans Streptococci, Streptococcus mutans, Bacillus spp., Micrococcus, and Enterococci were investigated.

SPSS 20 software (SPSS Inc., Chicago, IL, USA) was used for statistical analyses. Frequency percentage was used for data description, and the mean descriptive in dex and standard deviation (SD) were used to report the average number of colonies at each department. Chi-square, McNemar, and Kruskal-Wallis tests were used to compare of contamination among different departments, to compare the frequency of contamithe frequency nation before and after clinical practice, and to compare the number of colonies, respectively. The significance level was considered less than 0.05 .

\section{Results:}

In the current study, 15 units of surgical, pediatrics, prosthodontics, endodontics, and restorative dentistry departments were evaluated in terms of microbial contamination on different surfaces. According to the results of the McNemar test, there is a statistically significant difference in the contamination frequency before and after dental treatment $(\mathrm{P}<0.001)$. Also, the highest number of contaminated samples after treatment was related to prosthodontics and restorative dentistry departments. The second-highest number of contaminated samples belonged to endodontics and pediatrics departments, and the lowest number of contaminated samples belonged to the surgical department. This difference in the number of contaminated samples was statistically significant $(\mathrm{P}=0.008)$.

Tables 1 and 2 show the average number of colonies of different evaluated species on different surfaces and in different departments.

Table 1. Mean number of microorganism colonies (CFU) by the sampled surfaces before and after clinical practice

\begin{tabular}{|c|c|c|c|c|c|c|c|c|}
\hline & \multicolumn{2}{|c|}{ Unit headrest } & \multicolumn{2}{|c|}{ Lamp handle } & \multicolumn{2}{|c|}{ Dental seat } & \multicolumn{2}{|c|}{ Air } \\
\hline & & $\mathrm{n} \pm \mathrm{SD}$ & & $\mathrm{a} \pm \mathrm{SD}$ & & $\mathrm{n} \pm \mathrm{SD}$ & & $\pm \mathrm{SD}$ \\
\hline \multicolumn{5}{|c|}{$S D=$ Standard Deviation, $C F U=$ Colony-Forming Unit } & & & & \\
\hline & Before & After & Before & After & Before & After & Before & After \\
\hline $\begin{array}{c}\text { Number of } \\
\text { microorganisms }\end{array}$ & $0.67 \pm 1.29$ & $1.67 \pm 1.54$ & $0.67 \pm 0.61$ & $1.60 \pm 0.82$ & $1.13 \pm 1.18$ & $2.33 \pm 1.63$ & $1.53 \pm 1.80$ & $6.60 \pm 3.97$ \\
\hline
\end{tabular}




\section{N Hoshyari, et al,}

Table 2. Mean number of microorganism colonies (CFU) by the departments before and after clinical practice

\begin{tabular}{|c|c|c|c|c|c|c|c|c|c|c|c|c|}
\hline \multirow{3}{*}{ Departments } & \multicolumn{3}{|c|}{ Unit headrest } & \multicolumn{3}{|c|}{ Lamp handle } & \multicolumn{3}{|c|}{ Dental seat } & \multicolumn{3}{|c|}{ Air } \\
\hline & \multicolumn{2}{|c|}{ Mean \pm SD } & \multirow[t]{2}{*}{ P-Value } & \multicolumn{2}{|c|}{ Mean \pm SD } & \multirow[t]{2}{*}{ P-Value } & \multicolumn{2}{|c|}{ Mean \pm SD } & \multirow[t]{2}{*}{ P-Value } & \multicolumn{2}{|c|}{ Mean \pm SD } & \multirow[t]{2}{*}{ P-Value } \\
\hline & Before & After & & Before & After & & Before & After & & Before & After & \\
\hline \multirow[t]{2}{*}{ Surgery } & $0.33 \pm 0.57$ & $1.00 \pm 1.73$ & 0.31 & $0.33 \pm 0.57$ & $0.67 \pm 1.15$ & 0.31 & $1.33 \pm 1.52$ & $2.00 \pm 2.64$ & 0.31 & $4.33 \pm 2.08$ & $11.00 \pm 1.0$ & 0.10 \\
\hline & & & & & & & & & & & 0 & \\
\hline Pediatrics & $0.33 \pm 0.57$ & $1.33 \pm 0.57$ & 0.08 & $0.67 \pm 0.57$ & $1.67 \pm 0.57$ & 0.08 & $0.67 \pm 1.15$ & $1.33 \pm 1.52$ & 0.15 & $0.33 \pm 0.57$ & $5.00 \pm 2.64$ & 0.10 \\
\hline Prosthodontics & $0.33 \pm 0.57$ & $2.00 \pm 1.00$ & 0.10 & $0.67 \pm 0.57$ & $2.00 \pm 0.00$ & 0.10 & $1.00 \pm 0.00$ & $2.00 \pm 0.00$ & 0.08 & $1.00 \pm 0.00$ & $5.33 \pm 1.52$ & 0.10 \\
\hline Endodontics & $0.33 \pm 0.57$ & $1.00 \pm 1.00$ & 0.15 & $1.33 \pm 0.57$ & $2.00 \pm 1.00$ & 0.15 & $1.33 \pm 0.57$ & $3.33 \pm 1.52$ & 0.10 & $0.33 \pm 0.57$ & $2.00 \pm 1.73$ & 0.10 \\
\hline $\begin{array}{c}\text { Restorative } \\
\text { dentistry }\end{array}$ & $2.00 \pm 2.64$ & $3.00 \pm 2.64$ & 0.08 & $0.33 \pm 0.57$ & $1.67 \pm 0.57$ & 0.10 & $1.33 \pm 2.30$ & $3.00 \pm 1.73$ & 0.10 & $1.67 \pm 1.15$ & $9.67 \pm 4.04$ & 0.10 \\
\hline
\end{tabular}

$S D=$ Standard Deviation, $C F U=$ Colony-Forming Unit

The results of these tables showed that after treatment, the highest rate of contamination was related to air, dental seats, headrests, and lamp handles, respectively.

Table 3 shows different types of microbes present in the air that had the highest prevalence in our study.
The most common microorganisms found in the air samples before and after treatment were Staphylococcus epidermis and Staphylococcus aureus, respectively. Table 4 shows the frequency of microorganisms among all samples collected from the air and unit surfaces. Among all the samples, Staphylococcus aureus had the highest prevalence after treatment.

Table 3. Frequency of microorganisms in the air before and after clinical practice

\begin{tabular}{ccc}
\hline Microorganisms & Before & After \\
\hline Staphylococcus aureus & Frequency (\%) & Frequency (\%) \\
\hline Staphylococcus epidermidis & $5(33.3 \%)$ & $12(80 \%)$ \\
\hline Staphylococcus saprophyticus & $3(20.0 \%)$ & $14(93.3 \%)$ \\
\hline viridans Streptococci & & $4(26.7 \%)$ \\
\hline Streptococcus mutans & $1(6.7 \%)$ & $3(20.0 \%)$ \\
\hline Bacillus spp. & & $3(20.0 \%)$ \\
\hline Micrococcus & $0(0.0 \%)$ & $9(60.0 \%)$ \\
\hline & $1(6.7 \%)$ & $3(20.0 \%)$ \\
\hline & $0(0.0 \%)$ & $0(0.0 \%)$ \\
\hline
\end{tabular}


Table 4. Frequency of microorganisms on all the sampled surfaces before and after clinical practice

\begin{tabular}{ccc}
\hline Microorganisms & Before & After \\
\hline & $\begin{array}{c}\text { Frequency } \\
(\%)\end{array}$ & Frequency $(\%)$ \\
\hline Staphylococcus aureus & $11(18.3 \%)$ & $42(70.0 \%)$ \\
\hline Staphylococcus epidermidis & $9(15.0 \%)$ & $33(55.0 \%)$ \\
\hline Staphylococcus saprophyticus & $24(40.0 \%)$ & $10(16.7 \%)$ \\
\hline viridans Streptococci & $1(1.7 \%)$ & $7(11.7 \%)$ \\
\hline Streptococcus mutans & $0(0.0 \%)$ & $3(5.0 \%)$ \\
\hline Bacillus spp. & & \\
\hline Micrococcus & $2(3.3 \%)$ & $11(18.3 \%)$ \\
\hline & $0(0.0 \%)$ & $5(8.3 \%)$ \\
\hline & & $3(5.0 \%)$ \\
\hline
\end{tabular}

\section{Discussion:}

Infection control is an important and noteworthy category because of its close relationship with dental care. ${ }^{(6)}$ Given the presence of contagious diseases such as hepatitis, acquired immunodeficiency syndrome (AIDS), and hospital infections, dental workplaces can be an environment for disease transmission. ${ }^{(7)}$ Therefore, inappropriate disinfection of dental environments can be a threat to the health of dental staff and patients.

Given the high probability of surface contamination in dentistry, the dispersion of aerosols and their potential to spread contamination, and the importance of these materials in cross-contamination, the present study was conducted to determine the prevalence of microbial contamination in clinical work surfaces at Sari Dental School.

In the present study, after examining the units in different departments, it was ob- served that work surface contamination at the end of dental work was higher than that before treatment. This finding is consistent with the findings reported by Valian et al, Khorakian et al, and Williams et al. ${ }^{(5,8,9)}$

Also, in the present study, the highest rate of contamination after treatment for all samples (surfaces and the air) belonged to Staphylococci, followed by Streptococci.

The results of the study by Valian et al showed that the most common types of bacteria after treatment are Staphylococci and Streptococci, ${ }^{(5)}$ which is consistent with the findings of the present study. Also, in the study by Khorakian et al, high contamination with Staphylococcus aureus and Bacillus spp. was reported on some surfaces, ${ }^{(8)}$ which is in line with the findings of the current study. However, in a study by Abbasi et al, the highest contamination rate reported on radiographic equipment belonged to Micrococcus $(75.7 \%),{ }^{(10)}$ 
which is not consistent with the findings of the present study.

In the current study, nutrient agar plates were used to study aerosols and air contamination. The results of the present study showed that Staphylococci, Streptococci, Bacillus spp., and Micrococcus were found in air samples. The highest number of colonies found in the air belonged to Staphylococcus aureus, followed by Bacillus spp.

In a study on measuring air contamination during dental surgery, Monarca et al used an air sampler with various plates near the dental assistant at a distance of about 1.5 meters from the patient to determine the total number of bacteria, fungi, Staphylococci, and Streptococcus. ${ }^{(2)}$ The results of the mentioned study indicated high air contamination, and all the samples contained Streptococcus and Staphylococcus, ${ }^{(2)}$ which is consistent with the findings of the current study.

In a study by Rautemaa et al, the density of aerobic bacterial contamination at a distance less than 1 meter from the patient was examined. ${ }^{(11)}$ The results of the cited study showed that the highest contamination rate belonged to gram-positive cocci including viridans Streptococcus and Staphylococci.

In the present study, the level of contamination of unit headrests, lamp handles, dental seats, and the air surrounding the unit was investigated. Different studies have examined different surfaces. Khorakian et al investigated the level of contamination of unit headrests, lamp handles, and dental seats, ${ }^{(8)}$ similar to the surfaces examined in the current study. These parts exist in all departments, and patients and dentists are in contact with these parts. In the study by Valian et al, the samples were collected from headrests, lamp handles, and tray handles. ${ }^{(5)}$ In a study by Smith et al, contamination levels of dental handpieces were investigated. ${ }^{(12)}$ Anjumn et al investigated the contamination of laptop keyboards in clinical departments of the Faculty of Dentistry. ${ }^{(13,14)}$ more contaminated than the units of the periodontics department. ${ }^{(5)}$ The duration of treatment in restorative dentistry department and use of highspeed turbines with cooling water spray may justify this result. Taking multiple oral impressions in prosthodontics departments and students' lack of attention to disinfection protocols can be the reasons for the higher contamination rate in the mentioned departments.

In the study by Khorakian et al, various microorganisms were examined, and the highest rate of Staphylococcus aureus was reported in the prosthodontics department, followed by pediatrics, periodontics, orthodontics, and endodontics departments. ${ }^{(8)}$ The findings of the present study are consistent with the findings reported by Khorkian et al. (8) In a study by Mahdavian et al regarding the contamination in prosthodontics department of Mashhad Faculty of Dentistry, the highest level of contamination belonged to Bacillus, followed by Staphylococcus aureus, and the highest level of contamination was observed on lamp handles and unit handles. ${ }^{(15)}$

\section{Conclusion:}

The results of the present study showed that Staphylococci were more prevalent on the examined surfaces, and a significant difference was found in the frequency of contamination before and after clinical practice. There was no significant difference in the total number of microorganisms among different departments after clinical practice. Bacterial contamination of the air was greater than other parts, followed by dental seat contamination.

\section{Acknowledgments:}

The present article has been derived from thesis No. 1697, approved by Mazandaran University of Medical Sciences. We wish to express our sincere gratitude to all people who have contributed to this thesis. 


\section{References:}

Umar D, Basheer B, Husain A, Baroudi K, Ahamed F, Kumar A. Evaluation of bacterial contamination in a clinical environment. J Int Oral Health. 2015;7(1):53.

2.Monarca S, Grottolo M, Renzi D, Paganelli C, Sapelli P, Zerbini I, et al. Evaluation of environmental bacterial contamination and procedures to control cross infection in a sample of Italian dental surgeries. Occup Environ Med. 2000 Nov;57(11):721-6.

3.Bennett A, Fulford M, Walker J, Bradshaw D, Martin M, Marsh P. Occupational health: Microbial aerosols in general dental practice. Br Dent J. 2000;189(12):664-7.

4.Prospero E, Savini S, Annino I. Microbial aerosol contamination of dental healthcare workers' faces and other surfaces in dental practice. Infect Control Hospital Epidemiol. 2003;24(2):139-41.

5.Valian A, Shahbazi R, Farshidnia S, Tabatabaee FS. Evaluation of the bacterial contamination of dental units in restorative and periodontics departments of Dental School of Shahid Beheshti University of Medical Sciences. J Mash Dent Sch. 2014;37(4):345-56.

6.Cleveland JL, Bonito AJ, Corley TJ, Foster M, Barker L, Brown G, et al. Advancing infection control in dental care settings: factors associated with dentists' implementation of guidelines from the Centers for Disease Control and Prevention. J Am Dent Assoc. 2012 Oct;143(10):1127-38.

7.Decraene V, Ready D, Pratten J, Wilson M. Air-borne microbial contamination of surfaces in a UK dental clinic. J Gen Appl Microbiol. 2008;54(4):195-203.

8.Khorakian F, Movahed T, Ghazvini K, Karbasi S, Tabrizi Nouri S, Bahramian L, et al. Evaluation of Frequency of Microbial Contamination in Clinical Setting Surface in Dental School of Mashhad University of Medical Sciences. J Mash Dent Sch. 2017;41(3):209-18.

9.Williams HN, Singh R, Romberg E. Surface contamination in the dental operatory: A comparison over two decades. J Am Dent Assoc. 2003;134(3): 325-30.

10.Abbasi F, Eslami G, Ghaem Maghami A. Prevalence of gram positive cocci contamination in the water lines of Shahid Beheshti Dental School units and drinking water supply of local area. Shahid Beheshti Univ Dent J. 2005;23(2):256-63.

11.Rautemaa R, Nordberg A, WuolijokiSaaristo K, Meurman JH. Bacterial aerosols in dental practice - a potential hospital infection problem? J Hosp Infect. 2006;64(1):76-81 .
12.Smith A, Smith G, Lappin DF, Baxter HC, Jones A, Baxter RL. Dental handpiece contamination: a proteomics and surface analysis approach. Biofouling. 2014 Jan;30(1):29-39.

13. Mostafavi A, Koosha S, Amjad M. Effect of Disinfection on the Surface Roughness of Dental Casts Retrieved from Addition Silicone Impressions. J Res Dentomaxillofac Sci. 2018;3(1):27-33.

14.Anjumn MS, Reddy PP, Abbas I, Monica M, Rao Y. Microbial contamination of laptop keyboards in dental settings. Int J Pub Health Dent. 2012;2(2):4-6.

15.Mahdavian SJ, Ghanaat J, Sadeghi J, Rahimi M. A study of bacterial contamination control of dental units in Prosthodontic department of Mashhad Dental School. J Mash Dent Sch. 2000;24(1-2):72-63.

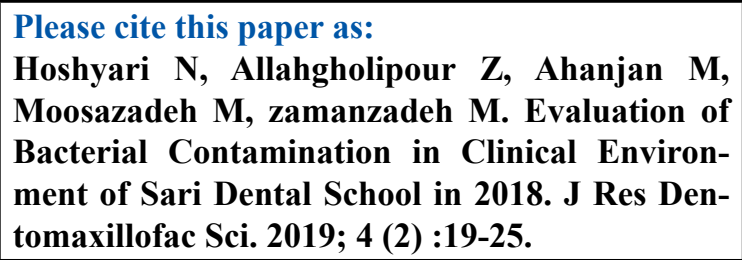

